\title{
Cauchy Six-Dimensional Formalism for Lamb Waves in Multilayered Plates
}

\author{
Sergey V. Kuznetsov \\ Institute for Problems in Mechanics, Russian Academy of Sciences, 101 Prospekt Vernadskogo, Moscow 129526, Russia \\ Correspondence should be addressed to Sergey V. Kuznetsov; kuzn-sergey@yandex.ru
}

Received 29 May 2013; Accepted 23 July 2013

Academic Editors: J. Clayton, C. J. Ho, and J.-I. Jang

Copyright (c) 2013 Sergey V. Kuznetsov. This is an open access article distributed under the Creative Commons Attribution License, which permits unrestricted use, distribution, and reproduction in any medium, provided the original work is properly cited.

Propagation of Lamb waves in multilayered elastic anisotropic plates is studied in the framework of combination of the sixdimensional Cauchy formalism and the transfer matrix method. The closed form secular equations for dispersion curves of Lamb waves propagating in multilayered plates with arbitrary elastic anisotropy are obtained.

\section{Introduction}

Herein, a brief introduction to the theory of Lamb waves and a review of some of the most important works on this matter are presented.

1.1. Lamb Waves in a Homogeneous Isotropic Plate. The first works $[1,2]$ on waves propagating in an infinite isotropic homogeneous plate with the traction-free boundary surfaces were done at the assumption that the wavelength is much longer than the plate thickness.

The complete theory of harmonic Lamb waves free from the long wavelength limit assumption was presented in [3]. The starting point of the Lamb theory is considering the equation of motion in the form

$$
c_{P} \nabla \operatorname{div} \mathbf{u}-c_{S} \operatorname{rot} \operatorname{rot} \mathbf{u}=\frac{\partial^{2} \mathbf{u}}{\partial t^{2}},
$$

where $\mathbf{u}$ is the displacement field and $c_{P}$ and $c_{S}$ are velocities of the longitudinal and transverse bulk waves, respectively:

$$
c_{P}=\sqrt{\frac{\lambda+2 \mu}{\rho}}, \quad c_{S}=\sqrt{\frac{\mu}{\rho}} .
$$

In (2), $\lambda$ and $\mu$ are Lamé constants and $\rho$ is the material density. Then, the displacement field was represented in terms of scalar $(\Phi)$ and vector $(\Psi)$ potentials

$$
\mathbf{u}=\nabla \Phi+\operatorname{rot} \Psi .
$$

The potentials were assumed to be harmonic in time:

$$
\Phi(\mathbf{x}, t)=\Phi^{\prime}(\mathbf{x}) e^{i \omega t}, \quad \Psi(\mathbf{x}, t)=\Psi^{\prime}(\mathbf{x}) e^{i \omega t} .
$$

Substituting representation (4) into (1) yields two independent Helmholtz equations:

$$
\left(\Delta+\frac{\omega^{2}}{c_{P}^{2}}\right) \Phi^{\prime}=0, \quad\left(\Delta+\frac{\omega^{2}}{c_{S}^{2}}\right) \Psi^{\prime}=0 .
$$

To define the spatial periodicity and to simplify the analysis, the splitting spatial argument is needed:

$$
\mathbf{x}=(\mathbf{x} \cdot \mathbf{n}) \mathbf{n}+(\mathbf{x} \cdot \boldsymbol{v}) \boldsymbol{v}+(\mathbf{x} \cdot \mathbf{w}) \mathbf{w}
$$

where $\mathbf{n}$ is the unit wave vector, $\boldsymbol{v}$ is the unit normal to the median plane of the plate, and $\mathbf{w}=\mathbf{n} \times \boldsymbol{v}$.

Remark 1. For the considered waves, it was further assumed that the displacement field does not depend upon argument $\mathbf{x} \cdot \mathbf{w}$. That allowed Lamb [3] to consider scalar potentials $\Psi$ and $\Psi^{\prime}$ in (4) instead of vector ones (actually, Lamb considered the vector potential composed of one nonvanishing component $(\Psi \cdot \mathbf{w}) \mathbf{w})$.

The further assumption relates to the periodicity of the potentials in the direction of propagation

$$
\Phi^{\prime}(\mathbf{x})=\varphi\left(x^{\prime \prime}\right) e^{x^{\prime}}, \quad \Psi^{\prime}(\mathbf{x})=\psi\left(x^{\prime \prime}\right) e^{x^{\prime}},
$$


where the dimensionless complex coordinates $x^{\prime}$ and $x^{\prime \prime}$ are

$$
x^{\prime}=\operatorname{ir} \mathbf{x} \cdot \mathbf{n}, \quad x^{\prime \prime}=i r \mathbf{x} \cdot \boldsymbol{\nu} .
$$

In (8), $i=\sqrt{-1}$ and $r$ is the wave number related to the wavelength $l$ by

$$
r=\frac{2 \pi}{l}
$$

Substituting representations (7) into (5) results in the decoupled ordinary differential equations

$$
\frac{d^{2} \varphi}{d x^{\prime \prime 2}}+\left(1-\frac{c^{2}}{c_{P}^{2}}\right) \varphi=0, \quad \frac{d^{2} \psi}{d x^{\prime \prime 2}}+\left(1-\frac{c^{2}}{c_{S}^{2}}\right) \psi=0,
$$

where the phase speed $c$ relates to the frequency and the wave number by the following relation:

$$
c=\frac{\omega}{r} .
$$

The general solution of (10) can be written in the form

$$
\begin{aligned}
& \varphi\left(x^{\prime \prime}\right)=C_{1} \sinh \left(\gamma_{1} x^{\prime \prime}\right)+C_{2} \cosh \left(\gamma_{1} x^{\prime \prime}\right), \\
& \psi\left(x^{\prime \prime}\right)=C_{3} \sinh \left(\gamma_{2} x^{\prime \prime}\right)+C_{4} \cosh \left(\gamma_{2} x^{\prime \prime}\right),
\end{aligned}
$$

where

$$
\gamma_{1}=\left(1-\frac{c^{2}}{c_{P}^{2}}\right)^{1 / 2}, \quad \gamma_{2}=\left(1-\frac{c^{2}}{c_{S}^{2}}\right)^{1 / 2}
$$

The unknown coefficients in (12) are defined (up to a multiplier) from the following boundary conditions on the free surfaces:

$$
\begin{gathered}
\mathbf{t}_{\nu} \equiv\left(\lambda \operatorname{tr}(\nabla \mathbf{u}) \mathbf{I}+\mu\left(\nabla \mathbf{u}+\nabla \mathbf{u}^{t}\right)\right) \cdot \boldsymbol{\nu}=0, \\
\mathbf{x} \cdot \boldsymbol{\nu}= \pm h
\end{gathered}
$$

where $2 h$ is the depth of the plate. Substitution representation (3) into boundary conditions (14) yields boundary conditions written in terms of potentials $\varphi^{\prime \prime}$ and $\psi^{\prime \prime}$ :

$$
\begin{gathered}
\left(\lambda \Delta \Phi^{\prime} \mathbf{I}+2 \mu\left(\nabla \nabla \Phi^{\prime}+\frac{1}{2}\left(\nabla \operatorname{rot} \Psi^{\prime}\right.\right.\right. \\
\left.\left.\left.+\left(\nabla \operatorname{rot} \Psi^{\prime}\right)^{t}\right)\right)\right) \cdot \boldsymbol{\nu}=0, \\
\mathbf{x} \cdot \boldsymbol{v}= \pm h .
\end{gathered}
$$

Substituting solutions (12) into (15), in view of Remark 1, yields the desired dispersion equation, found in $[1,3]$ :

$$
\frac{\tanh \left(\gamma_{2} r h\right)}{\tanh \left(\gamma_{1} r h\right)}-\left(\frac{4 \gamma_{1} \gamma_{2}}{\left(1+\gamma_{2}^{2}\right)^{2}}\right)^{ \pm 1}=0
$$

The sign “+” refers to symmetrical and “-” to antisymmetrical modes. In view of expressions (11) and (13), the obtained dispersion equation implicitly determines the phase velocity $c$ as a function of frequency. Equations for velocities related to the long wave and short wave limits were found in [3].

Taking the short wave limit at $r h \rightarrow \infty$ in (16) yields

$$
1=\left(\frac{4 \gamma_{1} \gamma_{2}}{\left(1+\gamma_{2}^{2}\right)^{2}}\right)
$$

The latter expression coincides with the secular equation for the speed of Rayleigh waves derived in [4], and hence the first limiting speed coincides with the speed of Rayleigh wave

$$
c_{1, \lim }=c_{R}
$$

Analysis of (16) at $r h \rightarrow 0$ (the long wave limit) yields the following equation [5]:

$$
\frac{\gamma_{2}}{\gamma_{1}}=\left(\frac{4 \gamma_{1} \gamma_{2}}{\left(1+\gamma_{2}^{2}\right)^{2}}\right)
$$

from where the values for two limiting velocities can be obtained:

$$
\begin{gathered}
c_{2, \lim }=2 c_{S} \sqrt{1-\frac{c_{S}^{2}}{c_{p}^{2}} .} \\
c_{3, \lim }=c_{S} .
\end{gathered}
$$

Expression (20) coincides with the limiting velocity value found in $[1,2]$ and differs from the anticipated value for the long wave limiting velocity of the sound waves in rods.

For the antisymmetric fundamental mode, (16) was analytically solved in [6] using the perturbation technique. Earlier studies of the dispersion curves for Lamb waves in a layer contacting with a half-space were mainly associated with the geophysical applications [7-10]. The analysis of the dispersion curves at different Poisson's ratios (including negative values) was done in [11-13]. The points of intersection of the dispersion curves were studied in [14].

1.2. Group Velocity. Consider now the notion of the group velocity introduced by Stokes [15] for description of the wave package propagation in hydrodynamics and later extrapolated to acoustic waves in the theory of elasticity; see [16-18]. Formally, the group velocity can be defined by

$$
c^{\text {group }}=\frac{d \omega}{d r}
$$

Numerical studies [19-25] of the group velocity dispersion, mainly at Poisson's condition $\lambda=\mu$, confirmed Rayleigh's anticipation $[16,17]$ that the negative values of the group velocity can appear at the very small wave numbers. Later on, numerical computations [25] revealed the existence of a broader range of negative group velocities at Poisson's ratios belonging to the interval $0.31<v<0.45$. 
Several additional equations for computing dispersion of the group velocity can be constructed from (16). For example, substituting the phase speed defined by (11) into (13), denoting the left-hand side of (16) by $F^{ \pm}(r, \omega)$, and assuming that $\omega$ is a function of $r$, the derivative of (16) with respect to $r$ takes the form

$$
\frac{\partial F^{ \pm}(r, \omega)}{\partial r}+\frac{\partial F^{ \pm}(r, \omega)}{\partial \omega} \frac{d \omega}{d r}=0
$$

from where, in view of (22), the secular equation for the group speed takes the form

$$
c^{\text {group }}=-\frac{\left(\partial F^{ \pm}(r, \omega)\right) / \partial r}{\left(\partial F^{ \pm}(r, \omega)\right) / \partial \omega} .
$$

In (24), $r$ is considered as a function of $\omega$. Theoretical studies of the phase, group, and ray velocities were done in [26].

\subsection{Homogeneous Anisotropic Plates: Three-Dimensional For-} malism. In the first works on Lamb waves propagating in anisotropic plates, a three-dimensional formalism was used. Initially, that formalism was developed in [27] for analysis of Rayleigh waves in an anisotropic half-space; later on, this method was applied to half-spaces with different groups of elastic symmetry [28-32]. With necessary modification, the approach [27] was used for analyzing Lamb waves in anisotropic plates [33-43].

All these publications, except [33] where a more complicated case of a cylindrically anisotropic plate was considered, actually exploited the following representation for the displacement field:

$$
\mathbf{u}(\mathbf{x}, t)=(\sum_{k=1}^{6} C_{k} \underbrace{\mathbf{m}_{k} e^{\gamma_{k} x^{\prime \prime}}}_{\mathbf{u}_{k}\left(x^{\prime \prime}\right)}) e^{i(r \mathbf{n} \cdot \mathbf{x}-\omega t)}
$$

where $C_{k}$ are arbitrary complex coefficients determined up to a multiplier by satisfying conditions at the plate boundaries, $\mathbf{u}_{k}$ is the displacement field of the $k$ th partial wave, $\mathbf{m}_{k}$ is a vectorial, generally, complex amplitude, determined by the Christoffel equation (this equation will be introduced later on), and $\gamma_{k}$ is a root of the Christoffel equation. Note that, according to (8), the coordinate $x^{\prime \prime}$ is imaginary. Six partial waves in (25) correspond to six (not necessary aliquant) roots of the Christoffel equation.
Substituting representation (25) into equation of motion

$$
\mathbf{A}\left(\partial_{x}, \partial_{t}\right) \mathbf{u} \equiv \operatorname{div}_{x} \mathbf{C} \cdot \nabla_{x} \mathbf{u}-\rho \ddot{\mathbf{u}}=0,
$$

where $\mathbf{C}$ is the fourth-order elasticity tensor assumed to be positive definite, yields the Christoffel equation

$$
\left[\left(\gamma_{k} \boldsymbol{v}+\mathbf{n}\right) \cdot \mathbf{C} \cdot\left(\mathbf{n}+\gamma_{k} \boldsymbol{\nu}\right)-\rho c^{2} \mathbf{I}\right] \cdot \mathbf{m}_{k}=0
$$

where $\mathbf{I}$ is the unit diagonal matrix. Equation (27) admits the equivalent form

$$
\operatorname{det}\left[\left(\gamma_{k} \boldsymbol{\nu}+\mathbf{n}\right) \cdot \mathbf{C} \cdot\left(\mathbf{n}+\gamma_{k} \boldsymbol{\nu}\right)-\rho c^{2} \mathbf{I}\right]=0
$$

The left-hand side of (28) represents a polynomial of degree six with respect to the Christoffel parameter $\gamma_{k}$. Equations (27), (28) show that roots $\gamma_{k}$ and the corresponding eigenvectors $\mathbf{m}_{k}$ can be considered as functions of the phase speed c.

Remark 2. (a) For Rayleigh waves, the roots $\gamma_{k}$ in representation (25) should be complex with $\operatorname{Im}\left(\gamma_{k}\right)<0$; this ensures attenuation of Rayleigh wave in the "lower" half-space $(\boldsymbol{v} \cdot \mathbf{x})<$ 0 . The condition $\operatorname{Im}\left(\gamma_{k}\right)<0$ confines the admissible speed interval and decreases the number of summation terms in (25). If $\operatorname{Re}\left(\gamma_{k}\right)=0$ for all partial waves composing Rayleigh wave, then such a wave is called the genuine Rayleigh wave; if $\operatorname{Re}\left(\gamma_{k}\right) \neq 0$ for some $k$, then such a wave is called the generalized Rayleigh wave [28]. For Lamb waves, the cases $\operatorname{Re}\left(\gamma_{k}\right)=0$ and $\operatorname{Re}\left(\gamma_{k}\right) \neq 0$ are usually not distinguished.

(b) Within the discussed formalism, the case of appearing multiple roots $\gamma_{k}$ and the coincident kernel eigenvectors $\mathbf{m}_{k}$ was considered in [44] with application to Rayleigh waves and in [45] for obtaining the dispersion equation of subsonic Lamb waves.

(c) For anisotropic plates, the limiting velocities (20), (21) were computed in $[46,47]$.

To obtain the dispersion equation, consider the tractionfree boundary conditions

$$
\left.\mathbf{t}_{\nu}\right|_{\mathbf{X} \cdot \boldsymbol{v}= \pm h} \equiv \pm\left.\boldsymbol{v} \cdot \mathbf{C} \cdots \nabla_{\mathbf{X}} \mathbf{u}\right|_{\mathbf{X} \cdot \boldsymbol{v}= \pm h}=0,
$$

where $2 h$ denotes the overall thickness of the plate. Substituting representation (25) into the boundary conditions (29) yields the dispersion equation in the form

$$
\sum_{k=1}^{6} C_{k}\left(\gamma_{k} \boldsymbol{\nu} \cdot \mathbf{C} \cdot \boldsymbol{v}+\boldsymbol{v} \cdot \mathbf{C} \cdot \mathbf{n}\right) \cdot \mathbf{m}_{k} e^{ \pm i r \gamma_{k} h}=0
$$

Finally, (30) can be rewritten in a form better suited for numerical computations:

$$
\operatorname{det}\left(\begin{array}{llll}
\left(\gamma_{1} \boldsymbol{\nu} \cdot \mathbf{C} \cdot \boldsymbol{\nu}+\boldsymbol{\nu} \cdot \mathbf{C} \cdot \mathbf{n}\right) \cdot \mathbf{m}_{1} e^{+i r \gamma_{1} h} & \ldots\left(\gamma_{6} \boldsymbol{\nu} \cdot \mathbf{C} \cdot \boldsymbol{v}+\boldsymbol{\nu} \cdot \mathbf{C} \cdot \mathbf{n}\right) \cdot \mathbf{m}_{6} e^{+i r \gamma_{6} h} \\
\left(\gamma_{1} \boldsymbol{\nu} \cdot \mathbf{C} \cdot \boldsymbol{v}+\boldsymbol{\nu} \cdot \mathbf{C} \cdot \mathbf{n}\right) \cdot \mathbf{m}_{1} e^{-i r \gamma_{1} h} & \ldots\left(\gamma_{6} \boldsymbol{\nu} \cdot \mathbf{C} \cdot \boldsymbol{v}+\boldsymbol{\nu} \cdot \mathbf{C} \cdot \mathbf{n}\right) \cdot \mathbf{m}_{6} e^{-i r \gamma_{6} h}
\end{array}\right)=0
$$


The dispersion equation (31) defines the phase speed as a function of the wave number or, in view of (11), as a function of the circular frequency.

1.4. Homogeneous Anisotropic Plates: Stroh Six-Dimensional Formalism. Initially, Stroh formalism [48] was applied to analysis of Rayleigh waves propagating on a free boundary of an anisotropic half-space [49-55]. The case of the nonsemisimple degeneracy of the fundamental matrix was considered in [53] (that case is associated with appearing multiple roots and the coincident kernel eigenvectors in the Christoffel equation). In $[56,57]$, the Stroh formalism was applied to the description of Lamb waves propagating in the homogeneous anisotropic plates.

Following [49], the displacement field for Rayleigh or Lamb wave is searched in the form

$$
\mathbf{u}(\mathbf{x}, t)=\mathbf{f}\left(x^{\prime \prime}\right) e^{i(r \mathbf{n} \cdot \mathbf{x}-\omega t)}
$$

with the unknown amplitude $\mathbf{f}$ regarded as a function of the imaginary coordinate $x^{\prime \prime}$ defined by (8). Substituting representation (32) into equation of motion (26) yields

$$
\left(\mathbf{A}_{1} \partial_{x^{\prime \prime}}^{2}+\left(\mathbf{A}_{2}+\mathbf{A}_{2}^{t}\right) \partial_{x^{\prime \prime}}+\mathbf{A}_{3}\right) \cdot \mathbf{f}\left(x^{\prime \prime}\right)=0,
$$

where

$$
\mathbf{A}_{1}=\boldsymbol{v} \cdot \mathbf{C} \cdot \boldsymbol{v}, \quad \mathbf{A}_{2}=\boldsymbol{v} \cdot \mathbf{C} \cdot \mathbf{n}, \quad \mathbf{A}_{3}=\mathbf{n} \cdot \mathbf{C} \cdot \mathbf{n}-\rho c^{2} \mathbf{I} .
$$

The matrix $\mathbf{A}_{1}$ is nondegenerate due to the assumption of positive definiteness of the elasticity tensor.

Remark 3. For an isotropic material, matrices (34) take the following form:

$$
\begin{aligned}
\mathbf{A}_{1} & =\left(\begin{array}{ccc}
\lambda+2 \mu & 0 & 0 \\
0 & \mu & 0 \\
0 & 0 & \mu
\end{array}\right), \quad \mathbf{A}_{2}=\left(\begin{array}{lll}
0 & \lambda & 0 \\
\mu & 0 & 0 \\
0 & 0 & 0
\end{array}\right), \\
\mathbf{A}_{3} & =\left(\begin{array}{ccc}
\mu-\rho c^{2} & 0 & 0 \\
0 & \lambda+2 \mu-\rho c^{2} & 0 \\
0 & 0 & \mu-\rho c^{2}
\end{array}\right) .
\end{aligned}
$$

Up to the harmonic multiplier $e^{i(r \mathbf{n} \cdot \mathbf{x}-\omega t)}$, the surface tractions on the planes parallel to the free boundary (or the median surface of a plate) can be written in terms of matrices (34):

$$
\mathbf{t}_{v}\left(x^{\prime \prime}\right) \equiv\left(\mathbf{A}_{1} \partial_{x^{\prime \prime}}+\mathbf{A}_{2}\right) \cdot \mathbf{f}\left(x^{\prime \prime}\right) .
$$

The main idea of Stroh formalism is in rewriting the equation of motion (26) in terms of the displacements and surface tractions. Multiplying both sides of (36) by matrix $\mathbf{A}_{1}^{-1}$ yields the following expression for the derivative $\partial_{x^{\prime \prime}} \mathbf{f}\left(x^{\prime \prime}\right)$ :

$$
\partial_{x^{\prime \prime}} \mathbf{f}\left(x^{\prime \prime}\right)=\mathbf{A}_{1}^{-1} \cdot \mathbf{t}_{v}\left(x^{\prime \prime}\right)-\mathbf{A}_{1}^{-1} \cdot \mathbf{A}_{2} \cdot \mathbf{f}\left(x^{\prime \prime}\right) .
$$

Combining now (33)-(37) produces the desired equation of motion written in terms of vectors $\mathbf{f}$ and $\mathbf{t}_{v}$ :

$$
\partial_{x^{\prime \prime}}\left(\begin{array}{c}
\mathbf{f} \\
\mathbf{t}_{v}
\end{array}\right)=\mathbf{N} \cdot\left(\begin{array}{c}
\mathbf{f} \\
\mathbf{t}_{v}
\end{array}\right)
$$

where $\mathbf{N}$ is the fundamental matrix [58]

$$
\mathbf{N}=\left(\begin{array}{cc}
-\mathbf{A}_{1}^{-1} \cdot \mathbf{A}_{2} & \mathbf{A}_{1}^{-1} \\
\mathbf{A}_{2}^{t} \cdot \mathbf{A}_{1}^{-1} \cdot \mathbf{A}_{2}-\mathbf{A}_{3} & -\mathbf{A}_{2}^{t} \cdot \mathbf{A}_{1}^{-1}
\end{array}\right)
$$

Now, the general solution of (38) can be represented in the form

$$
\left(\begin{array}{c}
\mathbf{f} \\
\mathbf{t}_{v}
\end{array}\right)=\exp \left(x^{\prime \prime} \mathbf{N}\right) \cdot \vec{C}_{6},
$$

where $\vec{C}_{6}$ is the six-dimensional generally complex vector of the unknown coefficients; that vector can be defined (up to a multiplier) by the boundary conditions. Substituting solution (40) into boundary conditions (29) yields

$$
\begin{aligned}
& \left(\begin{array}{c}
\mathbf{f}(+i r h) \\
\mathbf{0}
\end{array}\right)=\exp (+i r h \mathbf{N}) \cdot \vec{C}_{6} \\
& \left(\begin{array}{c}
\mathbf{f}(-i r h) \\
\mathbf{0}
\end{array}\right)=\exp (-i r h \mathbf{N}) \cdot \vec{C}_{6} .
\end{aligned}
$$

Excluding vector $\vec{C}_{6}$ from (41a) and substituting the resultant expression into (41b) gives the following dispersion equation for an anisotropic plate:

$$
\operatorname{det}((\mathbf{0}, \mathbf{I}) \cdot \exp (-2 i r h \mathbf{N})) \cdot\left(\begin{array}{l}
\mathbf{I} \\
\mathbf{0}
\end{array}\right)=0
$$

Despite the obvious simplicity of deriving (42), the relevant works on Lamb waves in anisotropic plates $[56,57]$ use a more complicated procedure.

1.5. Multilayered Plates: The Transfer Matrix Method. The transfer matrix method suggested for analyzing propagation of Lamb and Rayleigh waves multilayered isotropic media was introduced in $[59,60]$. Up to now, the transfer matrix method was combined with the three-dimensional formalism. The main idea of the method is to exclude the unknown coefficients $C_{k}$ in representation (25) expressing them in terms of the only partly known surface tractions and displacements on one of the outer surfaces of the plate; such a procedure is similar to (41a), (41b). If the plate contains several layers, the interface conditions can also be expressed in terms of these surface tractions and displacements via specially constructed transfer matrices. Finally, the boundary conditions on the other outer surface are expressed in terms of the surface tractions and displacements from the first outer surface.

Since its introduction, the transfer matrix method was applied to finding the dispersion relations for Lamb waves propagating in both isotropic [60-62] and monoclinic (or with higher symmetries) $[63,64]$ multilayered plates. Despite the abundance and simplicity, the original variant of 
the transfer matrix method revealed some numerical instability, especially when high frequencies and large depths of the layers were considered. To overcome this problem, the numerically stable algorithms were suggested [65-70]. Differing in details, these algorithms have the common principle idea of eliminating the terms containing large exponentials. Such a procedure, developed in [65], is known as the $\delta$-matrix method $[66,67]$. The $\delta$-matrix method can also be applied for analyzing dispersion of Lamb waves in a single-layered plate; see [65].

In the following sections, the transfer matrix method will be coupled with the Cauchy six-dimensional formalism, and a numerically stable approach resembling [65-70] will be worked out.

1.6. Multilayered Plates: The Global Matrix Method. The global matrix method introduced in [71] appeared more numerically stable than the original version of the transfer matrix method; see [62, 65] for discussions. In [72-77] applications of the global matrix method to analyses of Lamb wave dispersion are presented.

There are several variants of constructing the global matrix. For a traction-free plate containing $n$-homogeneous

anisotropic layers, the matrix equation related to the global matrix method can be written in the following form:

$$
\begin{aligned}
\overrightarrow{\mathbf{0}}_{6 n}= & \left(\begin{array}{ccccc}
(\mathbf{0}, \mathbf{I}) \cdot \mathbf{D}_{1}^{+} & & & & \\
\mathbf{D}_{1}^{-} & -\mathbf{D}_{2}^{+} & & & \\
& \mathbf{D}_{2}^{-} & -\mathbf{D}_{3}^{+} & & \\
& & \cdots & \cdots & \\
& & & & (\mathbf{0}, \mathbf{I}) \cdot \mathbf{D}_{n}^{-}
\end{array}\right) \\
& \cdot\left(\begin{array}{c}
\vec{C}_{1} \\
\vec{C}_{2} \\
\vec{C}_{3} \\
\vdots \\
\vec{C}_{n}
\end{array}\right),
\end{aligned}
$$

where $\vec{C}_{k} \in \mathbb{C}^{6}, k=1, \ldots, n$, are six-dimensional generally complex vectors containing the unknown coefficients in representation (25); $\mathbf{D}_{k}^{ \pm}$are $6 \times 6$-matrices defining displacements by (25) and surface tractions by (30) on both surfaces of a layer:

$$
\mathbf{D}_{k}^{ \pm}=\left(\begin{array}{ccc}
\mathbf{m}_{k_{1}} e^{ \pm i r \gamma_{k_{1}} h_{k}} & \cdots & \mathbf{m}_{k_{6}} e^{ \pm i r \gamma_{k_{6}} h_{k}} \\
\left(\gamma_{k_{1}} \boldsymbol{\nu} \cdot \mathbf{C}_{k} \cdot \boldsymbol{\nu}+\boldsymbol{\nu} \cdot \mathbf{C}_{k} \cdot \mathbf{n}\right) \cdot \mathbf{m}_{k_{1}} e^{ \pm i r \gamma_{k_{1}} h_{k}} & \cdots & \left(\gamma_{k_{6}} \boldsymbol{\nu} \cdot \mathbf{C}_{k} \cdot \boldsymbol{\nu}+\boldsymbol{\nu} \cdot \mathbf{C}_{k} \cdot \mathbf{n}\right) \cdot \mathbf{m}_{k_{6}} e^{ \pm i r \gamma_{k_{6}} h_{k}}
\end{array}\right) .
$$

Despite the reported numerical stability [72-74], the comparative study of the original transfer matrix method $[58,59]$ and the global matrix method [71] revealed that actually both methods exhibit some intrinsic instability resulting in loss of accuracy at high frequencies and large depths of the layers [78]. To overcome the persistent instability, in [78] a more refined rearrangement of the exponential terms, appearing in the global matrix method, was suggested.

\section{Cauchy Six-Dimensional Formalism}

In the following, another variant of a six-dimensional formalism for analyzing dispersion of Lamb waves in plates with arbitrary anisotropy is presented. Since the developed formalism is based on reduction of the second-order equation (33) to the Cauchy normal form, it will be called as the Cauchy formalism.

2.1. Basic Notations. For the considered formalism, the representation for harmonic Lamb wave is searched in the form (32). By introducing a new vector function

$$
\mathbf{v}\left(x^{\prime \prime}\right)=\partial_{x^{\prime \prime}} \mathbf{f}\left(x^{\prime \prime}\right)
$$

the equation of motion (26) can be reduced to the Cauchy normal form

$$
\partial_{x^{\prime \prime}}\left(\begin{array}{l}
\mathbf{f} \\
\mathbf{v}
\end{array}\right)=\mathbf{G} \cdot\left(\begin{array}{l}
\mathbf{f} \\
\mathbf{v}
\end{array}\right)
$$

where

$$
\mathbf{G}=\left(\begin{array}{cc}
0 & \mathbf{I} \\
-\mathbf{A}_{1} \cdot \mathbf{A}_{3} & -\mathbf{A}_{1} \cdot\left(\mathbf{A}_{2}+\mathbf{A}_{2}^{t}\right)
\end{array}\right)
$$

and matrices $\mathbf{A}_{1}, \mathbf{A}_{2}$, and $\mathbf{A}_{3}$ are defined by (34). By the analogy with Stroh formalism, $6 \times 6$-matrix $\mathbf{G}$ will be called the fundamental matrix.

Similar to (40), the general solution of (46) can be represented in Eulerian exponential form

$$
\left(\begin{array}{l}
\mathbf{f}\left(x^{\prime \prime}\right) \\
\mathbf{v}\left(x^{\prime \prime}\right)
\end{array}\right)=\exp \left(x^{\prime \prime} \mathbf{G}\right) \cdot \vec{C}_{6}
$$

where $\vec{C}_{6}$ is the six-dimensional generally complex vector of the unknown coefficients defined (up to a multiplier) by the boundary conditions.

The surface traction vector is defined by (36). Now, combining (48) and (36), the displacements and surface tractions on both sides of the plate take the form

$$
\left(\begin{array}{c}
\mathbf{f}( \pm i r h) \\
\mathbf{t}_{v}( \pm i r h)
\end{array}\right)=\mathbf{Z} \cdot \exp ( \pm i r h \mathbf{G}) \cdot \vec{C}_{6}
$$

where

$$
\mathbf{Z}=\left(\begin{array}{cc}
\mathbf{I} & \mathbf{0} \\
\mathbf{A}_{2} & \mathbf{A}_{1}
\end{array}\right)
$$

Note that matrix $\mathbf{Z}$ is nondegenerate due to positive definiteness of the elasticity tensor. 
Equation (49) allows us to exclude $\vec{C}_{6}$ by expressing the displacements and surface tractions on one side of the plate through the corresponding vectors on the other side; that yields

$$
\left(\begin{array}{c}
\mathbf{f}(-i r h) \\
\mathbf{t}_{v}(-i r h)
\end{array}\right)=\mathbf{T} \cdot\left(\begin{array}{c}
\mathbf{f}(+i r h) \\
\mathbf{t}_{v}(+i r h)
\end{array}\right)
$$

where

$$
\mathbf{T}=\mathbf{Z} \cdot \exp (-2 i r h \mathbf{G}) \cdot \mathbf{Z}^{-1} .
$$

Matrix $\mathbf{T}$ can be considered as the transfer matrix, as it "transfers" displacements and surface tractions from one side of the plate to another. It should be noted that matrix $\mathbf{T}$ is independent of boundary conditions.

2.2. Dispersion Relations for a Homogeneous Plate. Equation (51) is a source for constructing the dispersion relations and obtaining an expression for the limiting velocity $c_{2, \lim }$ at $r \rightarrow$ 0 .

2.2.1. Traction-Free Plate. If both sides of a plate are traction free, then

$$
\mathbf{t}_{v}( \pm i r h)=0 .
$$

Condition (53) means that a $3 \times 3$-mapping

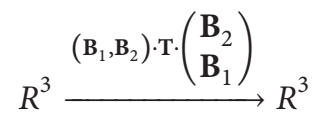

from the three-dimensional space of nontrivial displacements and vanishing surface-tractions on the "upper" surface to the three-dimensional space of the surface-tractions on the "bottom" surface is degenerate. In (54):

$$
\mathbf{B}_{1}=\mathbf{0}, \quad \mathbf{B}_{2}=\mathbf{I} \text {. }
$$

Matrices $\mathbf{B}_{1}$ and $\mathbf{B}_{2}$ are introduced for consistency with other types of boundary conditions that will be considered later in this section.

The condition (54) is equivalent to

$$
\operatorname{det}\left(\left(\mathbf{B}_{1}, \mathbf{B}_{2}\right) \cdot \mathbf{T} \cdot\left(\begin{array}{l}
\mathbf{B}_{2} \\
\mathbf{B}_{1}
\end{array}\right)\right)=0 .
$$

Thus, (56) is the desired dispersion equation for a plate with free boundaries.

Taking in (54) limit at $r \rightarrow 0$ does not lead to a meaningful condition, because of vanishing determinant in (56) at $r=0$ irrespective of the phase speed. To derive a meaningful equation, consider the first derivative of (54) with respect to the wave number (see [59-61]); this yields the following equation for $c_{2, \text { lim }}$ :

$$
\operatorname{det}\left(\left(\mathbf{B}_{1}, \mathbf{B}_{2}\right) \cdot \mathbf{Z} \cdot \mathbf{G} \cdot \mathbf{Z}^{-1} \cdot\left(\begin{array}{c}
\mathbf{B}_{2} \\
\mathbf{B}_{1}
\end{array}\right)\right)=0 .
$$

Remark 4. Substituting matrices (35) into (57) yields an isotropic plate (20).
2.2.2. Clamped Plate. If both sides of a plate are clamped, then

$$
\mathbf{f}( \pm i r h)=0
$$

Condition (58) means that mapping from the space of vanishing displacements and nontrivial surface tractions on the "upper" surface to the three-dimensional space of the displacements on the "bottom" surface is degenerate. But, this condition can be expressed by the same equations as (56) with the only difference in matrices $\mathbf{B}_{1}$ and $\mathbf{B}_{2}$ :

$$
\mathbf{B}_{1}=\mathbf{I}, \quad \mathbf{B}_{2}=\mathbf{0} .
$$

The equation for the limiting speed $c_{2, \lim }$ remains the same, as (57).

2.2.3. Plates with Mixed Boundary Conditions. Herein, two types of mixed boundary conditions at a boundary surface are considered: (i) vanishing normal surface-traction and vanishing tangential displacements and (ii) vanishing tangential surface-tractions and vanishing normal displacements. Boundary conditions (i) can be written in the form

$$
\boldsymbol{\nu} \cdot \mathbf{t}_{v}( \pm i r h)=0, \quad(\mathbf{I}-\boldsymbol{v} \otimes \boldsymbol{v}) \cdot \mathbf{f}( \pm i r h)=0 .
$$

For boundary conditions (60), matrices $\mathbf{B}_{1}$ and $\mathbf{B}_{2}$ become

$$
\mathbf{B}_{1}=\mathbf{I}-\boldsymbol{v} \otimes \boldsymbol{v}, \quad \mathbf{B}_{2}=\boldsymbol{v} \cdot \mathbf{t}_{v} .
$$

Similar to expressions (60), boundary conditions (ii) can be written in the form

$$
(\mathbf{I}-\boldsymbol{v} \otimes \boldsymbol{v}) \cdot \mathbf{t}_{v}( \pm i r h)=0, \quad \boldsymbol{v} \cdot \mathbf{f}( \pm i r h)=0,
$$

and $\mathbf{B}_{1}, \mathbf{B}_{2}$ become

$$
\mathbf{B}_{1}=\boldsymbol{v} \cdot \mathbf{t}_{v}, \quad \mathbf{B}_{2}=\mathbf{I}-\boldsymbol{v} \otimes \boldsymbol{v} .
$$

2.3. Dispersion Relations for a Multilayered Plate. Consider an $n$-layered plate with the homogeneous anisotropic layers. At the interfaces, ideal mechanical contact is assumed.

2.3.1. The Transfer Matrix Method. Assuming that propagation of the Lamb wave in each layer is determined by (45)(50) and writing a sequence of (51) that transfers boundary conditions from top to bottom yield

$$
\left(\begin{array}{c}
\mathbf{f}\left(-i r h_{n}\right) \\
\mathbf{t}_{v}\left(-i r h_{n}\right)
\end{array}\right)=\left(\prod_{k=1}^{n} \mathbf{T}_{k}\right) \cdot\left(\begin{array}{c}
\mathbf{f}\left(+i r h_{1}\right) \\
\mathbf{t}_{v}\left(+i r h_{1}\right)
\end{array}\right),
$$

where the lower index indicates the number of a layer and the transfer matrices $\mathbf{T}_{k}$ are defined by (52).

Now, multiplying both sides of (64) by matrices $\mathbf{B}_{1}, \mathbf{B}_{2}$, similar to (54)-(56) for the traction-free plate, we arrive at the dispersion equation for the multilayered traction-free plate:

$$
\operatorname{det}\left(\left(\mathbf{B}_{1}, \mathbf{B}_{2}\right) \cdot\left(\prod_{k=1}^{n} \mathbf{T}_{k}\right) \cdot\left(\begin{array}{l}
\mathbf{B}_{2} \\
\mathbf{B}_{1}
\end{array}\right)\right)=0 .
$$


Similar to (54), (65) reflects degeneracy of the mapping

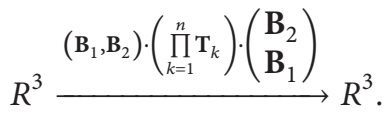

The dispersion equations for other types of boundary conditions have the same form as (65) but with obvious replacement of matrices $\mathbf{B}_{1}$ and $\mathbf{B}_{2}$.

As it was with (57), taking limit at $r \rightarrow 0$ of (65) does not lead to a meaningful condition, because of vanishing the determinant at $r=0$ irrespective of the phase speed. To derive a meaningful equation, consider the first derivative of the mapping (66) with respect to the wave number.

2.3.2. The Global Matrix Method. While the global matrix equation (43) mainly preserves its form, a minor modification is needed to account for other types of boundary conditions at the outer boundaries:

$$
\begin{aligned}
& \overrightarrow{\mathbf{0}}_{6 n}=\left(\begin{array}{ccccc}
\left(\mathbf{B}_{1}, \mathbf{B}_{2}\right) \cdot \mathbf{D}_{1}^{+} & & & & \\
\mathbf{D}_{1}^{-} & -\mathbf{D}_{2}^{+} & & & \\
& \mathbf{D}_{2}^{-} & -\mathbf{D}_{3}^{+} & & \\
& & \cdots & \cdots & \\
& & & & \left(\mathbf{B}_{1}, \mathbf{B}_{2}\right) \cdot \mathbf{D}_{n}^{-}
\end{array}\right) \\
& \left(\begin{array}{c}
\vec{C}_{1} \\
\vec{C}_{2} \\
\vec{C}_{3} \\
\vdots \\
\vec{C}_{n}
\end{array}\right)
\end{aligned}
$$

Within the considered Cauchy formalism, matrices $\mathbf{D}_{k}^{ \pm}$ change:

$$
\mathbf{D}_{k}^{ \pm}=\mathbf{Z}_{k} \cdot \exp \left( \pm i r h_{k} \mathbf{G}_{k}\right)
$$

where index $k$ indicates the corresponding layer.

\section{Numerical Examples}

3.1. Improving Numerical Stability of the Transfer Matrix Method. Performing numerical computations with the exponential matrices leads to numerical instability associated with either loss of precision or overflow due to the presence of the exponential terms with large positive powers.

3.1.1. The Overflow Problem. This problem can be relatively easily solved by a suitable normalization. Let the fundamental matrices $\mathbf{G}_{k, k=1, \ldots, n}$ be decomposed into their Jordan normal forms

$$
\mathbf{G}_{k}=\mathbf{W}_{k}^{-1} \cdot \mathbf{J}_{k} \cdot \mathbf{W}_{k}
$$

where $\mathbf{W}_{k}$ is a matrix composed of eigenvectors and possibly the generalized eigenvectors, while $\mathbf{J}_{k}$ is a matrix containing eigenvalues and possibly Jordan blocks if $\mathbf{G}_{k}$ is not a semisimple matrix.
With decomposition (69), the exponential matrices in (52) can be represented in the following form [79, 80]:

$$
\exp \left(-2 i r h_{k} \mathbf{G}_{k}\right)=\mathbf{W}_{k}^{-1} \cdot \exp \left(-2 i r h_{k} \mathbf{J}_{k}\right) \cdot \mathbf{W}_{k} \text {. }
$$

Let $\left(\lambda_{k}^{(m)}\right)_{m=1, \ldots, 6}$ denote (not necessary aliquant) eigenvalues of $\mathbf{G}_{k}$, and

$$
a=\underbrace{\max }_{k, m} \operatorname{Re}\left(-2 i r h_{k} \lambda_{k}^{(m)}\right) .
$$

Multiplying all the exponent matrices in (65) by $e^{-a}$ yields the desired normalization. Indeed, after multiplication, all the exponential terms in (70) will have powers with real parts not exceeding unity.

3.1.2. Loss of Precision. As reported in [78], the extension of the $\delta$-matrix approach [65] to anisotropic media [67-70] and the global matrix methods still manifest numerical instability, which is mainly due to loss of numerical precision. To overcome this problem, the following measures are suggested: (i) computing minimal eigenvalue of the resultant matrix (for both transfer and global matrix methods), instead of computing the corresponding determinants, and (ii) using longer mantissas instead of "double precision" computations.

While being more time consuming, the first measure allows one to avoid possible overlook of a root. For example, consider a typical case with the following diagonal matrix:

$$
\mathbf{D}=\operatorname{diag}(\varepsilon, \bar{\varepsilon}, \alpha),
$$

where $\varepsilon$ is a small (generally complex) eigenvalue associated with the root and $\alpha$ is a large eigenvalue. In the vicinity of the root the determinant of matrix (72) has no change of sign and, thus, can lead to overlooking of the root. Moreover, if $\alpha$ increases at approaching the root, the determinant may even increase, at least being not very close to the root.

Necessity to perform multiprecision computations can be demonstrated by considering the following matrix:

$$
\mathbf{D}=\left(\begin{array}{cc}
1+\varepsilon(t) & -1 \\
-1 & 1-\varepsilon(t)
\end{array}\right)
$$

where $|\varepsilon(t)|<10^{-15}$ is a small function of the argument $t$. It is obvious that computing the determinant with double precision accuracy gives zero, inspite of the applied numerical algorithm. However, analytical evaluation yields

$$
\operatorname{det} \mathbf{D}=\varepsilon^{2}(t),
$$

confirming necessity in multiprecision computations.

In numerical examples considered below mantissas from $\sim 25$ decimal digits (quadruple precision) to $\sim 1000$ decimal digits were used, depending upon the analyzed problem.

\subsection{Numerical Examples}

3.2.1. Homogeneous Plates with Cubic Symmetry. Three plates made of materials with cubic symmetry are considered, all 


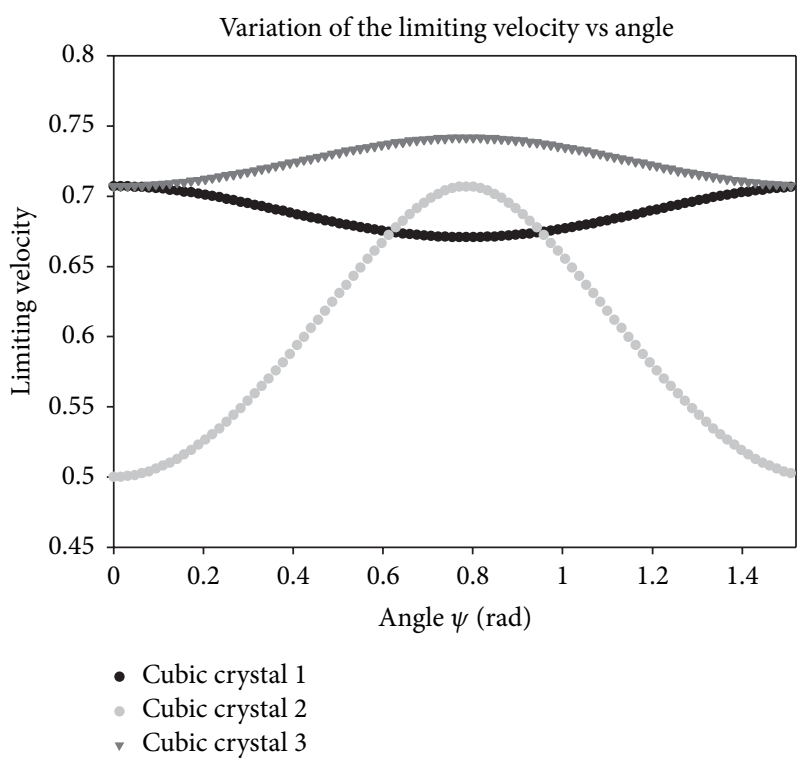

FIGURE 1: Variation of limiting velocity $c_{2, \lim }$ versus angle $\psi$.

with unit material density. To estimate deviation of elastic moduli of a cubic crystal from the isotropic elastic constants, the following measure can be introduced:

$$
\delta \equiv\left|\frac{C_{1111}}{C_{1122}+2 C_{1212}}-1\right| .
$$

In (75), $C_{1122}$ and $C_{1212}$ would correspond to Lamé parameters $\lambda$ and $\mu$, and $C_{1111}$ would be equal to the denominator in (75) if the material was isotropic.

The first considered crystal has elastic parameters that only slightly differ from the isotropic ones

$$
C_{1111}=1, \quad C_{1122}=0.1, \quad C_{1212}=0.5 .
$$

For the considered cubic crystal, the measuring parameter is relatively small: $\delta \approx 0.09$.

The second cubic crystal has the following elastic parameters:

$$
C_{1111}=1, \quad C_{1122}=0, \quad C_{1212}=0.25 .
$$

These parameters differ from the isotropic case in a much greater extent with $\delta=1$.

The third considered crystal belongs to a set of auxetic materials characterized by negative Poisson's ratios [81]:

$$
C_{1111}=1, \quad C_{1122}=-0.1, \quad C_{1212}=0.5 .
$$

For the auxetic crystal $(78), \delta \approx 0.09$, yielding the same value as for the first crystal.

For these crystals, vectors $\boldsymbol{v}$ and $\mathbf{n}$ have the following coordinates (relative to the principle elasticity directions):

$$
\boldsymbol{\nu}=(1 ; 0 ; 0), \quad \mathbf{n}=(0 ; \cos \psi ; \sin \psi) .
$$

Variation of the limiting velocity $\mathcal{c}_{2, \lim }$ with respect to angle $\psi$ is shown in Figure 1.
The plots in Figure 1 reveal that the limiting velocity $c_{2, \text { lim }}$ is fairly informative for identification of the propagation direction of the limiting Lamb wave: even for the first and third crystals, there are evident variations of $c_{2, \lim }$ values with respect to $\psi$.

Remark 5. In the NDT, the limiting Lamb wave propagating with the velocity $c_{2, \lim }$ plays a very important role, since such a wave can easily be excited. Indeed, energy needed for excitation is proportional to the square of the frequency, and for the considered limiting wave, the corresponding frequency vanishes.

3.2.2. Multilayered Plates. Herein, two multilayered tractionfree plates with alternating anisotropic layers are considered; the depth of each layer is $h=14 \mathrm{~nm}\left(14 \times 10^{-9} \mathrm{~m}\right)$. In the subsequent analysis, the direction of propagation of Lamb waves is $\mathbf{n}=(1,0,0)$ in coordinate axes oriented along principal directions of the elasticity tensors introduced below.

The alternating layers are made of hexagonal crystal SiC with $\rho=3220 \mathrm{~kg} / \mathrm{m}^{3}$ and the following elastic constants (in $\mathrm{GPa})[82]$ :

$$
\begin{gathered}
C_{1111}=503, \quad C_{1122}=109, \quad C_{1133}=52 \\
C_{2222}=503, \quad C_{2233}=52, \quad C_{3333}=552 \\
C_{2323}=C_{1313}=160, \quad C_{1212}=\frac{C_{1111}-C_{1122}}{2} ;
\end{gathered}
$$

the unit normals to planes of elastic isotropy are coincident with vector $\boldsymbol{v}$ (normal to the median plane of a layer). The second alternating layer is cubic crystal $\mathrm{Si}_{3} \mathrm{~N}_{4}(\gamma$-phase) with $\rho=2329 \mathrm{~kg} / \mathrm{m}^{3}$ and elastic constants (in GPa) [83]

$$
\begin{gathered}
C_{1111}=514, \quad C_{1122}=182, \quad C_{1133}=182 \\
C_{2222}=514, \quad C_{2233}=182, \quad C_{3333}=514 \\
C_{1212}=C_{1313}=C_{2323}=327 .
\end{gathered}
$$

Remark 6. It should be noted that the $\mathrm{SiC}$ crystal is piezoelectric, so in a more rigorous approach $[84,85]$ the change of elastic constants reflecting electrically open (constant electric displacement) or closed (constant electric field) circuit conditions should be foreseen.

The computed bulk wave velocities (in $\mathrm{m} / \mathrm{s}$ ) for the plane waves with normal $\mathbf{n}$ are

$$
\begin{gathered}
\text { SiC: } c_{P_{\mathrm{n}}}=12498 \quad c_{S_{\mathrm{n}}}{ }^{\text {in-plane }}=7822 \\
\mathcal{C}_{S_{\mathrm{n}}} \text { out of plane }=7049 \\
\mathrm{Si}_{3} \mathrm{~N}_{4}: c_{P_{\mathrm{n}}}=14856 \quad c_{S_{\mathrm{n}}}=11849 .
\end{gathered}
$$

For the considered multilayered plate, the dispersion curves for Lamb waves are plotted in Figure 2.

These plots were constructed by the described transfer matrix method in combination with the quadruple precision computations (mantissas with $\sim 34$ decimal digits). 


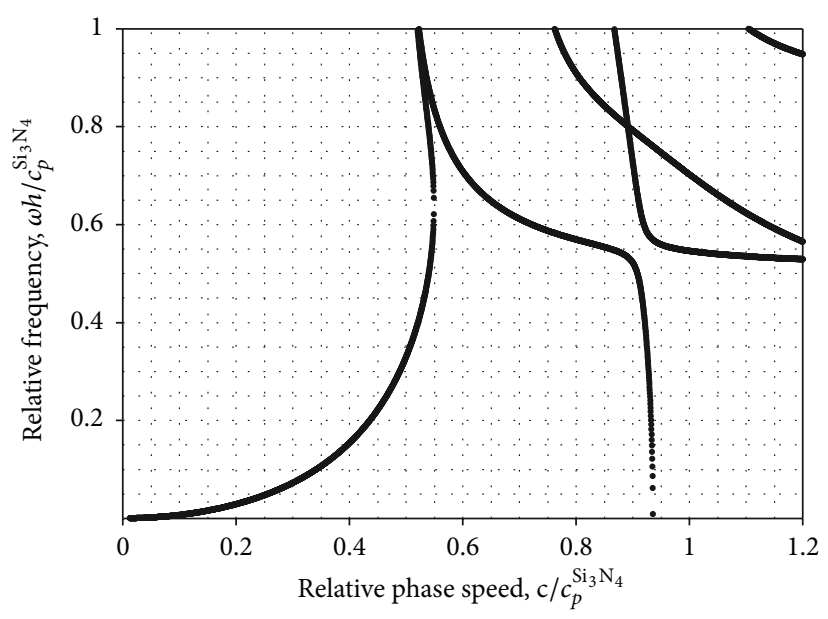

(a)

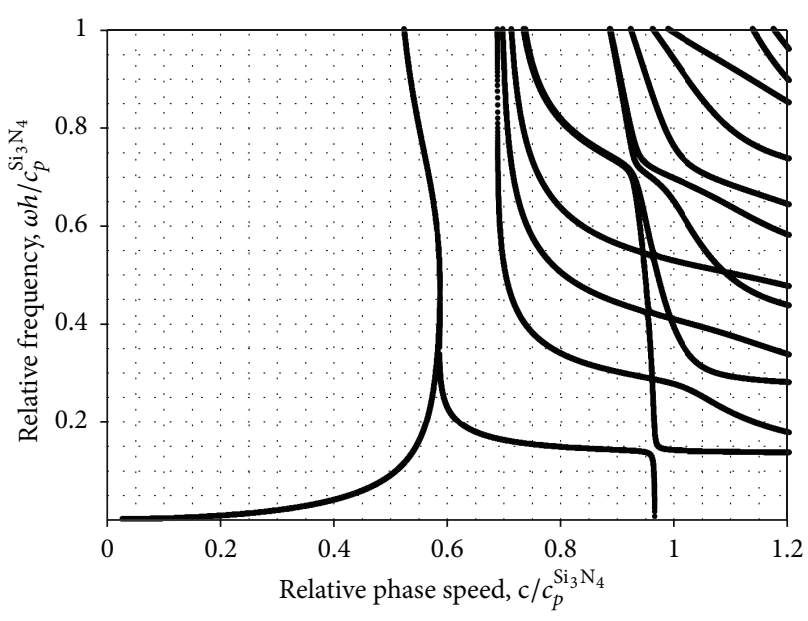

(b)

FIGURE 2: Dispersion curves for 3-layerd (a) and 11-layered (b) traction-free plates with alternating $\mathrm{SiC}-\mathrm{Si}_{3} \mathrm{~N}_{4}$ layers.

One of the most interesting observations concerns almost vertical parts of the dispersion curves at low-frequency limits; these are clearly seen in plots for both of the plates. The corresponding relative limiting velocity increases with increase of number of layers; however, the relative limiting velocity does not exceed unity, meaning that $c_{2, \text { lim }}<c_{P_{n}}^{\mathrm{Si}_{3} \mathrm{~N}_{4}}$. Another observation concerns the presence of the vertical asymptotes at large $\omega$ for lower branches. A more detailed analysis reveals that in both of these plates these asymptotes correspond to the same relative phase velocity $c_{R_{n}}^{\mathrm{SiC}} / c_{P_{n}}^{\mathrm{Si}_{3} \mathrm{~N}_{4}} \approx 0.497$, where $c_{R_{n}}^{\mathrm{SiC}}$ denotes velocity of Rayleigh wave in $\mathrm{SiC}$ for the considered propagation direction.

\section{Conclusion}

The Cauchy six-dimensional formalism is developed for analyzing propagation of Lamb waves in anisotropic multilayered plates. The presented numerical examples reveal its ability to obtain dispersion curves for plates with large number of anisotropic layers.

\section{References}

[1] J. W. Rayleigh, "On the free vibrations of an infinite plate of homogeneous isotropic elastic matter," Proceedings of the London Mathematical Society, vol. 20, pp. 225-234, 1889.

[2] H. Lamb, "On the flexure of an elastic plate," Proceedings of the London Mathematical Society, vol. 21, pp. 70-90, 1889.

[3] H. Lamb, "On waves in an elastic plate," Proceedings of the Royal Society A, vol. 93, pp. 114-128, 1917.

[4] J. W. Rayleigh, "On wave propagating along the plane surface of an elastic solid," Proceedings of the London Mathematical Society, vol. 17, pp. 4-11, 1885.

[5] R. D. Mindlin, "Waves and vibrations in isotropic, elastic plates," in Proceedings of the 1st Symposium on Naval Structural Mechanics, J. N. Goodies and N. J. Hoff, Eds., pp. 199-232, Pergamon Press, Oxford, UK.
[6] I. A. Viktorov, Rayleigh and Lamb Wave. Physical Theory and Application, Plenum Press, New York, NY, USA, 1967.

[7] H. Jeffreys, "The surface waves of Earthquakes," Geophysical Journal International, vol. 3, pp. 253-261, 1935.

[8] V. G. Gogoladze, "Dispersion of Rayleigh waves in a layer," Trudy Instituta Seismologii Akademii Nauk USSR, vol. 119, pp. 27-38, 1947 (Russian).

[9] J. T. Wilson and O. Baykal, "On the North Atlantic basin as determined from Rayleigh wave dispersion," Bulletin of the Seismological Society of America, vol. 38, pp. 41-53, 1948.

[10] M. B. Dobrin, "Dispersion in seismic surface waves," Geophysics, vol. 16, pp. 63-80, 1951.

[11] A. Freedmab, "The vibration, with the Poisson ratio, of Lamb modes in a free plate, I: general spectra," Journal of Sound and Vibration, vol. 137, no. 2, pp. 209-230, 1990.

[12] A. Freedman, "The variation, with the Poisson ratio, of Lamb modes in a free plate, II: at transitions and coincidence values," Journal of Sound and Vibration, vol. 137, no. 2, pp. 231-247, 1990.

[13] A. Freedman, "The variation, with the Poisson ratio, of Lamb modes in a free plate, III: behaviour of individual modes," Journal of Sound and Vibration, vol. 137, no. 2, pp. 249-266, 1990.

[14] Q. Zhu and W. G. Mayer, "On the crossing points of Lamb wave velocity dispersion curves," Journal of the Acoustical Society of America, vol. 93, no. 4 I, pp. 1893-1895, 1993.

[15] G. G. Stokes, "On the theory of oscillatory waves," Transactions of the Cambridge Philosophical Society, vol. 8, pp. 441-455, 1847.

[16] J. W. Rayleigh, "On progressive waves," Proceedings of the London Mathematical Society, vol. 9, pp. 21-26, 1877.

[17] J. W. Rayleigh, Theory of Sound, Ulan Press, 2011.

[18] O. Reynolds, "On the rate of progression of groups of waves and the rate at which energy is transmitted by waves," Nature, vol. 16, pp. 343-344, 1877.

[19] K. Sezawa and K. Kanai, "On the propagation of Rayleigh waves in dispersive elastic media," Bulletin of the Earthquake Research Institute, vol. 19, pp. 549-553, 1941.

[20] Y. Sato, "Study on surface waves. II: velocity of surface waves propagated upon elastic plates," Bulletin of the Earthquake Research Institute, vol. 29, pp. 223-261, 1951.

[21] F. Press and M. Ewing, "Theory of air-coupled flexural waves," Journal of Applied Physics, vol. 22, no. 7, pp. 892-899, 1951. 
[22] F. Press and J. Oliver, "Model study of air-coupled surface waves," Journal of the Acoustical Society of America, vol. 27, pp. 43-46, 1955.

[23] I. Tolstoy, "Dispersion and simple harmonic sources in wave ducts," Journal of the Acoustical Society of America, vol. 27, pp. 897-910, 1955.

[24] I. Tolstoy and E. Usdin, "Wave propagation in elastic plates: low and high mode dispersion," Journal of the Acoustical Society of America, vol. 29, pp. 37-42, 1957.

[25] K. Negishi, "Existence of negative group velocities for Lamb waves," Japanese Journal of Applied Physics, vol. 26, supplement 26-1, pp. 171-173, 1987.

[26] M. A. Biot, "General theorems on the equivalence of group velocity and energy transport," Physical Review, vol. 105, no. 4, pp. 1129-1137, 1957.

[27] R. Stoneley, "The seismological implications of aelotropy in continental structures," The Geophysical Supplements to Monthly Notices of the Royal Astronomical Society, vol. 5, pp. 343-353, 1949.

[28] M. J. P. Musgrave, "On the propagation of elastic waves in aeolotropic media. I. general principles," Proceedings of the Royal Society A, vol. 226, pp. 339-355, 1954.

[29] M. J. P. Musgrave, "On the propagation of elastic waves in aeolotropic media. II. media of hexagonal symmetry," Proceedings of the Royal Society A, vol. 226, pp. 356-366, 1954.

[30] V. T. Buchwald, "Rayleigh waves in transversely isotropic media," Quarterly Journal of Mechanics and Applied Mathematics, vol. 14, no. 3, pp. 293-318, 1961.

[31] R. Stoneley, "The propagation of surface elastic waves in a cubic crystal," Proceedings of the Royal Society A, vol. 232, pp. 447-458, 1955.

[32] G. F. Miller and M. J. P. Musgrave, "On the propagation of elastic waves in aeolotropic media. III. Media of cubic symmetry," Proceedings of the Royal Society A, vol. 236, pp. 352-383, 1956.

[33] M. Mitra, "Propagation of elastic waves in an infinite plate of cylindrically aelotropic material," Zeitschrift für angewandte Mathematik und Physik, vol. 10, no. 6, pp. 579-583, 1959.

[34] D. L. Anderson, "Elastic wave propagation in layered anisotropic media," Journal of Geophysical Research, vol. 66, pp. 2953-2963, 1961.

[35] L. P. Solie and B. A. Auld, "Elastic waves in free anisotropic plates," Journal of the Acoustical Society of America, vol. 54, pp. 50-65, 1973.

[36] A. H. Nayfeh and D. E. Chimenti, "Free wave propagation in plates of general anisotropic media," Journal of Applied Mechanics, vol. 56, no. 4, pp. 881-886, 1989.

[37] V. Dayal and V. K. Kinra, "Leaky Lamb waves in an anisotropic plate. I: an exact solution and experiments," Journal of the Acoustical Society of America, vol. 85, pp. 2268-2276, 1989.

[38] A. Ben-Menahem and A. G. Sena, "Seismic source theory in stratified anisotropic media," Journal of Geophysical Research, vol. 95, no. 10, pp. 15-427, 1990.

[39] G. R. Liu, J. Tani, K. Watanabe, and T. Ohyoshi, "Lamb wave propagation in anisotropic laminates," Journal of Applied Mechanics, vol. 57, pp. 923-9929, 1990.

[40] W. Lin and L. M. Keer, "A study of Lamb waves in anisotropic plates," Journal of the Acoustical Society of America, vol. 92, no. 2, pp. 888-894, 1992.

[41] G. Neau, Lamb waves in anisotropic viscoelastic plates. Study of the wave fronts and attenuation [Ph.D. thesis], Universite de Bordeaux, Bordeaux, France, 2003.
[42] G. Neau, M. Deschamps, and M. J. S. Lowe, "Group velocity of Lamb waves in anisotropic plates: comparison between theory and experiment," in Review of Progress in Quantitative NDE, D. O. Thompson and D. E. Chimenti, Eds., vol. 20, pp. 81-88, American Inst. Physics, New York, NY, USA, 2001.

[43] L. Wang and F. G. Yuan, "Group velocity and characteristic wave curves of Lamb waves in composites: modeling and experiments," Composites Science and Technology, vol. 67, no. 78, pp. 1370-1384, 2007.

[44] S. V. Kuznetsov, "Surface waves of non-Rayleigh type," Quarterly of Applied Mathematics, vol. 61, no. 3, pp. 575-582, 2003.

[45] S. V. Kuznetsov, "Subsonic lamb waves in anisotropic plates," Quarterly of Applied Mathematics, vol. 60, no. 3, pp. 577-587, 2002.

[46] S. A. Markus, "Low frequency approximations for zero-order normal modes in anisotropic plates," Akusticheskii Zhurnal, vol. 33, pp. 1091-1095, 1987 (Russian).

[47] V. I. Alshits and V. N. Lyubimov, "Abnormal dispersion of surface elastic waves in anisotropic plate," Kristallografiya, vol. 33, pp. 279-285, 1988 (Russian).

[48] A. N. Stroh, "Steady state problems in anisotropic elasticity," Journal of Mathematical Physics, vol. 41, pp. 77-103, 1962.

[49] K. A. Ingebrigtsen and A. Tonning, "Elastic surface waves in crystals," Physical Review, vol. 184, no. 3, pp. 942-951, 1969.

[50] D. M. Barnett and J. Lothe, "Synthesis of the sextic and the integral formalism for dislocations, Greens functions, and surface waves in anisotropic elastic solids," Physica Norvegica, vol. 7, pp. 13-19, 1973.

[51] P. Chadwick and G. D. Smith, "Foundations of the theory of surface waves in anisotropic elastic materials," Advances in Applied Mechanics, vol. 17, no. C, pp. 303-376, 1977.

[52] T. C. T. Ting and D. M. Barnett, "Classifications of surface waves in anisotropic elastic materials," Wave Motion, vol. 26, no. 3, pp. 207-218, 1997.

[53] T. C. T. Ting, "On extraordinary semisimple matrix N(v) for anisotropic elastic materials," Quarterly of Applied Mathematics, vol. 55, no. 4, pp. 723-738, 1997.

[54] Y. B. Fu, "Hamiltonian interpretation of the Stroh formalism in anisotropic elasticity," Proceedings of the Royal Society A, vol. 463, no. 2088, pp. 3073-3087, 2007.

[55] K. Tanuma, Stroh Formalism and Rayleigh Waves, Springer, New York, NY, USA, 2010.

[56] A. L. Shuvalov, "On the theory of wave propagation in anisotropic plates," Proceedings of the Royal Society A, vol. 456, pp. 2197-2222, 2000.

[57] V. I. Alshits, M. Deschamps, and G. A. Maugin, "Elastic waves in anisotropic plates: short-wavelength asymptotics of the dispersion branches $\nu \mathrm{n}(\mathrm{k}), "$ Wave Motion, vol. 37, no. 3, pp. 273-292, 2003.

[58] W. T. Thomson, "Transmission of elastic waves through a stratified solid medium," Journal of Applied Physics, vol. 21, no. 2, pp. 89-93, 1950.

[59] N. A. Haskell, "Dispersion of surface waves on multilayered media," Bulletin of the Seismological Society of America, vol. 4317, p. 34, 1953.

[60] N. Guo and P. Cawley, "Lamb wave propagation in composite laminates and its relationship with acousto-ultrasonics," NDT and E International, vol. 26, no. 2, pp. 75-84, 1993.

[61] D. E. Chimenti, "Lamb waves in microstructured plates," Ultrasonics, vol. 32, no. 4, pp. 255-260, 1994. 
[62] M. J. S. Lowe, "Matrix techniques for modeling ultrasonic waves in multilayered media," IEEE Transactions on Ultrasonics, Ferroelectrics, and Frequency Control, vol. 42, no. 4, pp. 525-542, 1995.

[63] A. H. Nayfeh, "The general problem of elastic wave propagation in multilayered anisotropic media," Journal of the Acoustical Society of America, vol. 89, no. 4 I, pp. 1521-1531, 1991.

[64] J. T. Stewart and Y.-K. Yong, "Exact analysis of the propagation of acoustic waves in multilayered anisotropic piezoelectric plates," IEEE Transactions on Ultrasonics, Ferroelectrics, and Frequency Control, vol. 41, no. 3, pp. 375-390, 1994.

[65] J. W. Dunkin, "Computation of modal solutions in layered elastic media at high frequencies," Bulletin of the Seismological Society of America, vol. 55, pp. 335-3358, 1965.

[66] D. Lévesque and L. Piché, "A robust transfer matrix formulation for the ultrasonic response of multilayered absorbing media," Journal of the Acoustical Society of America, vol. 92, no. 1, pp. 452-467, 1992.

[67] M. Castaings and B. Hosten, "Delta operator technique to improve the Thomson-Haskell-method stability for propagation in multilayered anisotropic absorbing plates," Journal of the Acoustical Society of America, vol. 95, no. 4, pp. 1931-1941, 1994.

[68] L. Wang and S. I. Rokhlin, "Stable reformulation of transfer matrix method for wave propagation in layered anisotropic media," Ultrasonics, vol. 39, no. 6, pp. 413-424, 2001.

[69] L. Wang and S. I. Rokhlin, "Modeling of wave propagation in layered piezoelectric media by a recursive asymptotic method," IEEE Transactions on Ultrasonics, Ferroelectrics, and Frequency Control, vol. 51, no. 9, pp. 1060-1071, 2004.

[70] E. L. Tan, "Hybrid compliance-stiffness matrix method for stable analysis of elastic wave propagation in multilayered anisotropic media," Journal of the Acoustical Society of America, vol. 119, no. 1, pp. 45-53, 2006.

[71] L. Knopoff, "A matrix method for elastic wave problems," Bulletin of the Seismological Society of America, vol. 54, pp. 431438, 1964

[72] A. K. Mal and L. Knopoff, "A differential equation for surface waves in layers with varying thickness," Journal of Mathematical Analysis and Applications, vol. 21, no. 2, pp. 431-444, 1968.

[73] F. Schwab and L. Knopoff, "Surface waves on multilayered anelastic media," Bulletin of the Seismological Society of America, vol. 61, pp. 893-912, 1971.

[74] M. J. Randall, "Fast programs for layered half-space problems," Bulletin of the Seismological Society of America, vol. 57, pp. 12991315, 1967.

[75] H. Schmidt and F. B. Jensen, "A full wave solution for propagation in multilayered viscoelastic media with application to Gaussian beam reflection at fluid-solid interfaces," Journal of the Acoustical Society of America, vol. 77, pp. 813-825, 1985.

[76] A. K. Mal, "Wave propagation in layered composite laminates under periodic surface loads," Wave Motion, vol. 10, no. 3, pp. 257-266, 1988.

[77] B. Pavlakovic, M. Lowe, D. Alleyne, and P. Cawley, "Disperse: a general purpose program for creating dispersion curves," in Review of Progress in Quantitative Nondestructive Evaluation, D. O. Thompson and D. E. Chimenti, Eds., vol. 16, pp. 185-1192, Plenum Press, NY, NY, USA, 1997.

[78] A. Abo-Zena, "Dispersion function computations for unlimited frequency values," Geophysical Journal of the Royal Astronomical Society, vol. 58, pp. 91-105, 1979.

[79] P. Hartman, Ordinary Differential Equations, SIAM, New York, NY, USA, 2002.
[80] M. C. Pease III, Methods of Matrix Algebra, Academic Press, London, UK, 1965.

[81] C. Y. Guo and L. Wheeler, "Extreme Poisson's ratios and related elastic crystal properties," Journal of the Mechanics and Physics of Solids, vol. 54, no. 4, pp. 690-707, 2006.

[82] K. Kamitani, M. Grimsditch, J. C. Nipko, C.-K. Loong, M. Okada, and I. Kimura, "The elastic constants of silicon carbide: a Brillouin-scattering study of $4 \mathrm{H}$ and $6 \mathrm{H} \mathrm{SiC}$ single crystals," Journal of Applied Physics, vol. 82, no. 6, pp. 3152-3154, 1997.

[83] A. Zerr, G. Miehe, G. Serghiou et al., "Synthesis of cubic silicon nitride," Nature, vol. 400, no. 6742, pp. 340-342, 1999.

[84] J. D. Clayton, "Modeling nonlinear electromechanical behavior of shocked silicon carbide," Journal of Applied Physics, vol. 107, no. 1, Article ID 013520, 18 pages, 2010.

[85] J. D. Clayton, Nonlinear Mechanics of Crystals, Springer, Dordrecht, The Netherlands, 2011. 

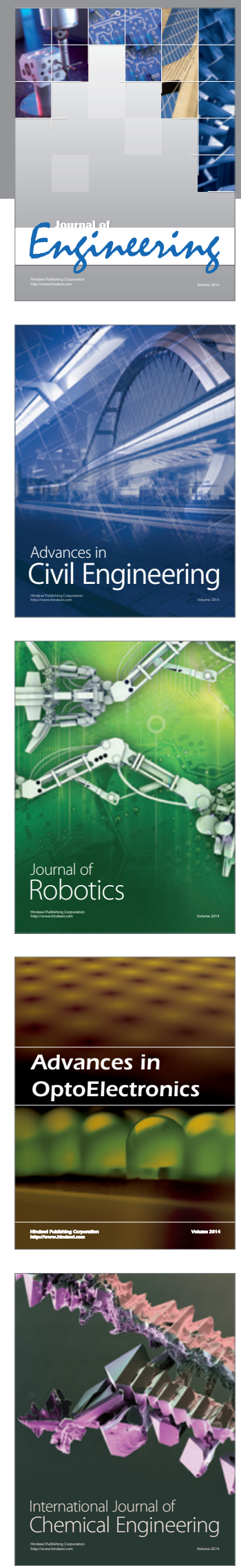

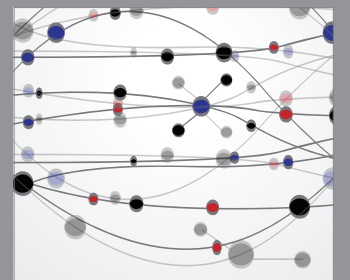

The Scientific World Journal
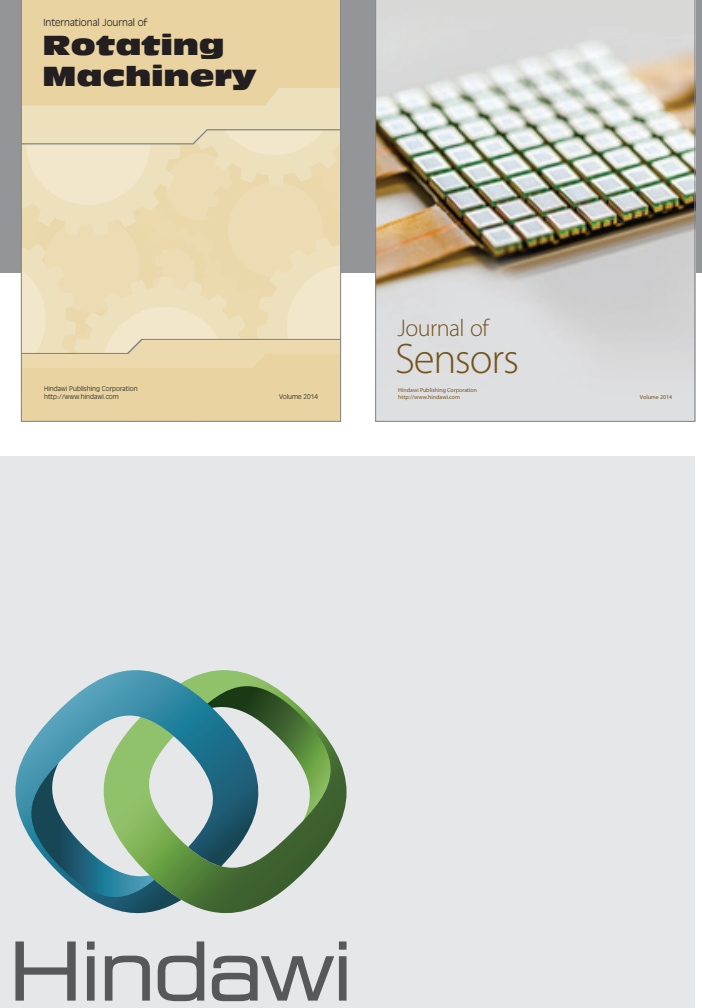

Submit your manuscripts at http://www.hindawi.com
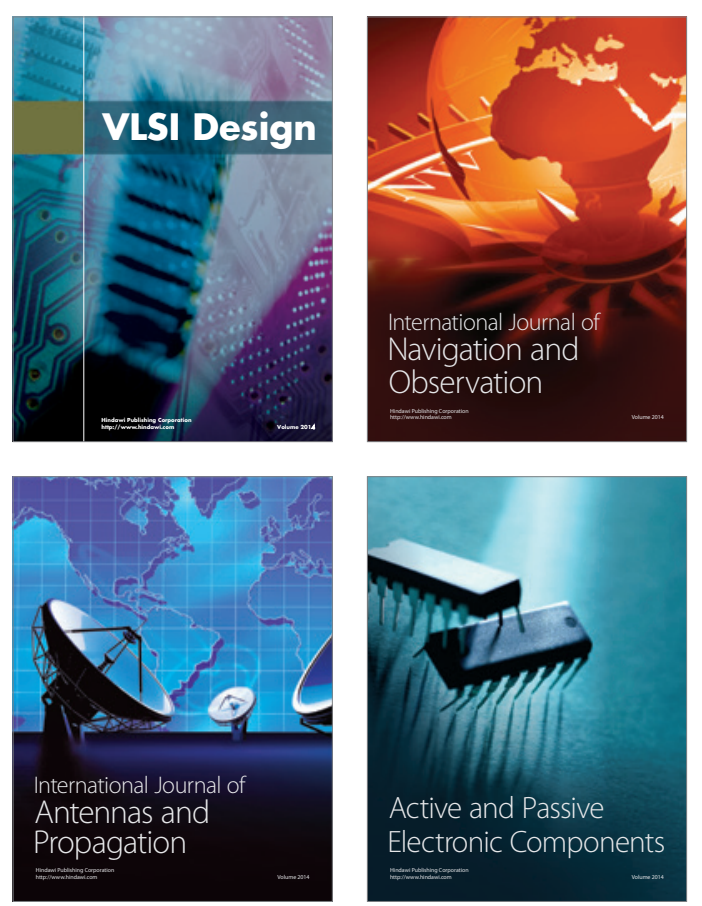
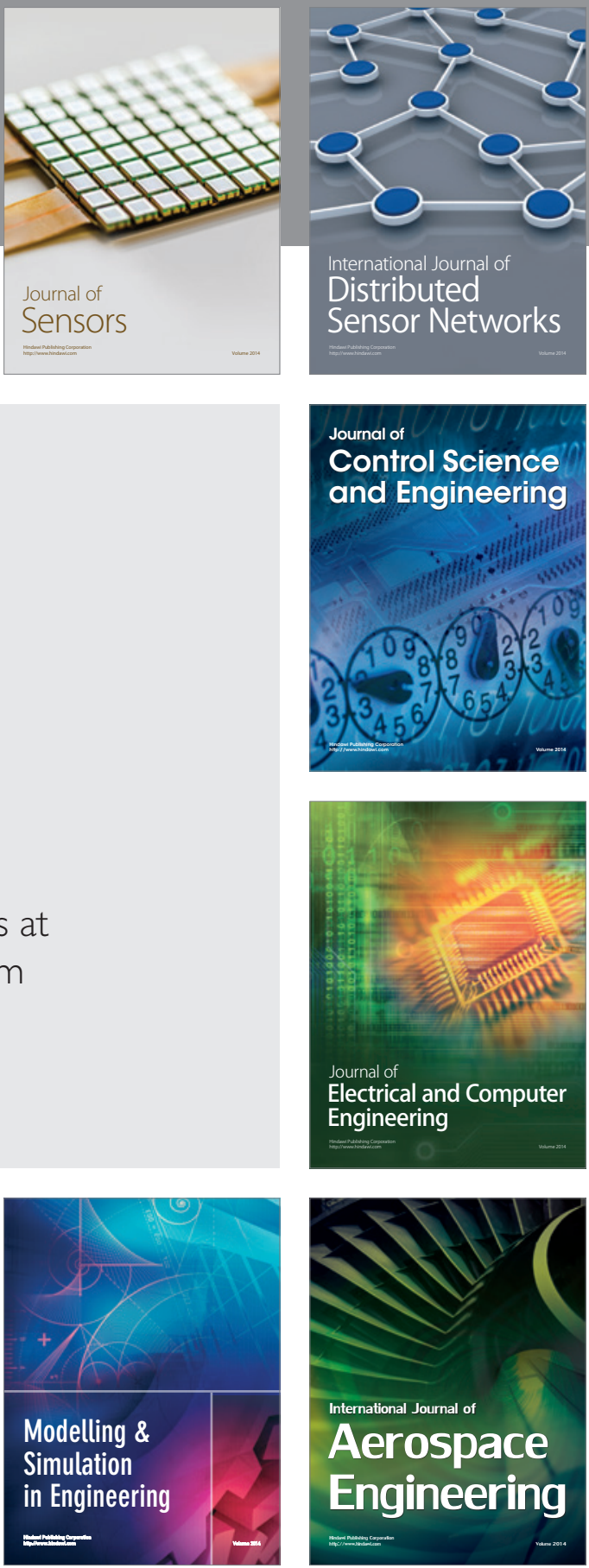

Journal of

Control Science

and Engineering
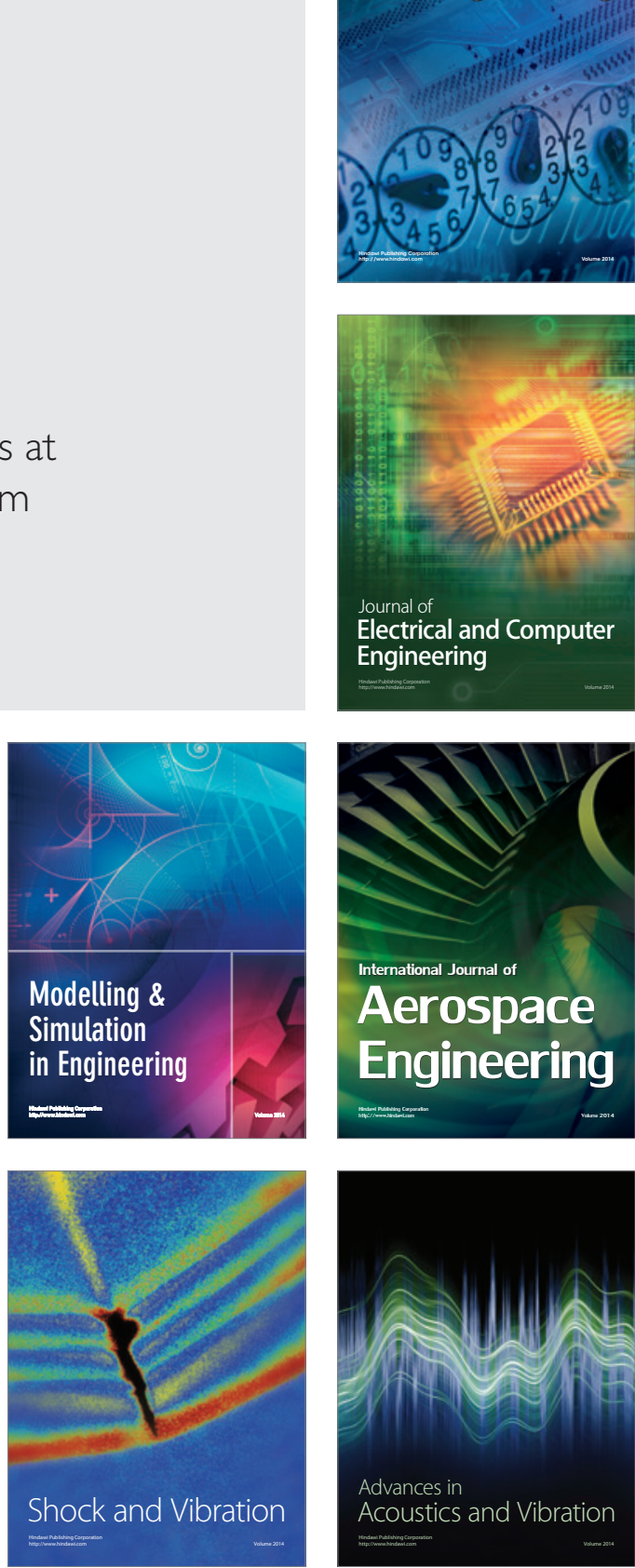